\title{
Magnetic Resonance \\ Cholangiopancreatography (MRCP) in the Evaluation of Pancreaticobiliary Tract in Gallstone Disease
}

\section{SN SINGH, TRILOK C BHATT}

\section{ABSTRACT}

Introduction: MRCP (Magnetic Resonance Cholangiopancreatography) has rapidly gained ground and has now firmly established in the evaluation of the biliary and pancreatic ducts. It often aids in the assessment of causes of biliary obstruction and can be helpful in the evaluation of the pancreatic duct without the inherent invasiveness of an endoscopic procedure.

In view of limitation of USG and CT and invasiveness of PTC, IVC and ERCP there is need for an imaging modality which is non invasive and provides high resolution projection images of the biliary and pancreatic duct.

Aim: The aim of this study was to prospectively assess the accuracy of MR imaging and correlate it with ultrasonography.

Materials and Methods: This study included 50 patients who had undergone MRCP prior to cholecystectomy for symptomatic gallstones. There were 23 males and 27 female patients, ranging in age from 8 to 87 years (mean, 63 years).

Results: The predominant population in our study was adults ranging from 30 to $50 \mathrm{yrs}$ of age. Majority of patients presented with epigastic pain and vomiting. The percentage rate of detection of gallstones and CBD stones were $89.75 \%$ and $76.19 \%$ on USG respectively. The same for MRCP was $87.18 \%$ and $95.24 \%$ respectively. MRCP showed high positive predictive value in diagnosing choledocholithaisis and diagnosing hepatobiliary lesions.

Conclusion: MRCP now readily permits the study and evaluation of anatomy and pathology of the pancreaticobiliary tree including pancreatic duct very easily and is accurate, non-invasive and non-ionizing imaging method of pancreaticobiliary anatomy and pathology. Our results demonstrate that preoperative MRCP had a robust safe guarding effect on laparoscopic cholecystectomy and deserves further promotion. These results were also sited in other recent studies.

Keywords: Choledocholithaisis, Cholelithiasis, Common bile duct (CBD),Ultrasonography

\section{INTRODUCTION}

Evaluation of suspected biliary obstruction has traditionally involved a variety of imaging modalities including ultrasonography (US), computed tomography (CT) and invasive cholangiography. These techniques have limitations because of poor visualization of intraductal stones on US and CT and the need for invasive procedures like ERCP and PTC. MRCP is a non-invasive imaging modality that provides good visualization of the hepato biliary system [1, 2].

Ultrasound is the initial screening tool that is used in evaluating patients presenting with pancreatic biliary diseases and was being mainly supplemented with CT [3]. Ultrasonography has limitations especially in the evaluation of the distal CBD where bowel gas, debris, fluid in the duodenum and obesity can degrade the image quality. CT scan has its share of limitations, especially in demonstrating two important pathologies, biliary stones and biliary strictures. CT has a sensitivity of only $90 \%$ for detecting biliary stones $[3,4]$.

Stones, which have high cholesterol and mixed stones may be difficult to detect on CT. Biliary strictures are not directly visualized on CT. The length and extent of the stricture is difficult to determine on CT. It is very important from a management point of view to visualize the length and extent of strictures. For these reasons cholangiographic modalities, intravenous cholangiography (IVC), percutaneous transhepatic cholangiography (PTC) and Endoscopic Retrograde Cholangiopancreatography (ERCP) are required. IV cholangiography has limitations, in $30-40 \%$ of cases there is incomplete opacification of the biliary system $[5,6]$. 
PTC and ERCP have the same diagnostic and therapeutic role but PTC is more invasive and risky with incidence of sepsis as high as $4 \%[7]$

In view of limitation of US and CT and invasiveness of PTC, IVC and ERCP there is need for an imaging modality which is non invasive and provides high resolution projection images of the biliary and pancreatic duct $[7,8]$.

With the development of higher magnetic field strength and newer pulse sequences, MRCP with its inherent high contrast resolution, rapidity, multiplanar capability and virtually artifact free display of anatomy and pathology in this region is proving to be examination of choice in patients with pancreatico biliary diseases [8].

This imaging technique is able to create projectile type images similar in detail an appearance to direct cholangiography. It avoids the use of IV contrast and ionizing radiation and is relatively operator independent. Several recent studies have demonstrated that MRCP is capable to accurately identify common bile duct stones with sensitivity of 81-100\%. Biliary strictures can also be visualized with sufficient anatomic detail to determine the level of obstruction and in some instances, differentiate benign from malignant causes [7]. In addition, MRCP lacks the major complication rate of approximately $3 \%$ associated with ERCP such as sepsis, bleeding, bile leak and death. MRCP demonstrates more than just the biliary and pancreatic ducts. Its disadvantages include lack of visualization of the peripheral intrahepatic ducts and inability to provide endobiliary therapy $[7,8]$.

\section{MATERIALS AND METHODS}

This was a prospective study conducted at Asvini Hospital, Mumbai over a period of three years in 2010-13. This study included 50 patients who had undergone MRCP prior to cholecystectomy for symptomatic gallstones. There were 23 males and 27 female patients, ranging in age from 8 to 87 years (mean, 63 years).

Patients were selected on the basis of -

1. Clinical history and laboratory criteria suggestive of having cholelithiasis or choledocholithiasis.

2. Findings of cholelithiasis or choledocholithiasis on other imaging modalities especially ultrasound.

\section{Exclusion criteria}

No significant exclusion criteria was formulated however, we did not include patients who were not willing for surgery/other interventional procedures like ERCP or patient who did not consent for post surgical follow-up.

\section{Patient preparation}

Patients were instructed to be on a fat free diet for $24 \mathrm{hrs}$ and study was performed after over night fast. Oral negative contrast was given for improved visualization of biliary tract and pancreatic duct.

\section{Technique}

Sonography was performed on GE-Logic 500 USG machine. Original sonographic reports regarding the presence of gall stones/dilatation of the CBD (>8 $\mathrm{mm}$ in diameter) were used to calculate the accuracy of the sonographic examination.

The patients were orally administered $1 \mathrm{ml}$ of gadolinium mixed in $250 \mathrm{ml}$ of pineapple juice. This worked as a negative contrast for stomach and duodenum. MRCP was performed in all patients after one hour with a 1.5-T system (Horizon; GE Medical Systems) by using a phased-array multicoil. Initially, localizer images were obtained in the coronal and transverse planes by using a spoiled gradient-recalled sequence. Subsequently, thin-section T2-weighted images were obtained by using a single-shot half-Fourier rapidacquisition and relaxation sequence (RARE-single-shot fast spin-echo) with a 260-340 mm field of view. In addition, HASTE [9] and PACE sequences were studied. All of the above sequences are included among the routine sequences of MR cholangiographic examination at our institution.

\section{RESULTS}

In the study, the number of patients with symptoms of gallstones were, predominantly females. The predominant age of adult population range from 30 to 50 years. Majority of patient presented with epigastric pain and vomiting. In my study, 39 cases had intraluminal gall bladder calculi and 21 cases had choledocholithiasis. Out of these 21 cases, 16 cases had associated gall bladder stones. Most common lesion was cholelithiasis $(n=39)$ followed by choledocholithiasis $(n=21)$. A representative case of cholelithiasis with choledocholithiasis is shown as [Table/Fig-1].

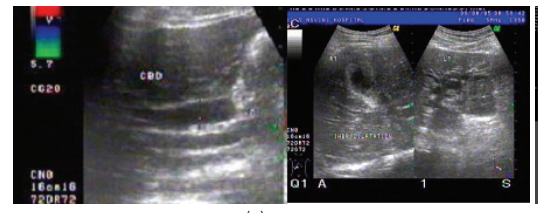

(a)

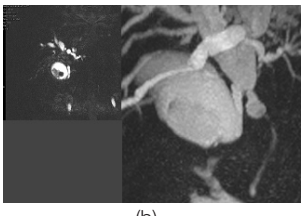

(b)
[Table/Fig-1]: Cholangiocarcinoma with distal choledocholithaisis (a) UGS - Grossly distended gall bladder (GB) with edematous wall \& a $11 \mathrm{~mm}$ calculus within (not shown). Moderate IHBR, CHD \& CBD (10 mm) dilatation. Distal CBD was not well delineated. (b) MRI confirmed findings of USG. Abrupt narrowing of mid third CBD with a $13 \mathrm{~mm}$ distal calculi. The strictured segment was Cholangiocarcinoma on HPA.

\section{DISCUSSION}

Transabdominal sonography is currently advocated for the initial evaluation of patients with symptoms consistent with choledocholithiasis. Currently Computed Tomography (CT) scanning is not the investigation of choice in evaluation of pancreaticobiliary tract and is recommended only for the 


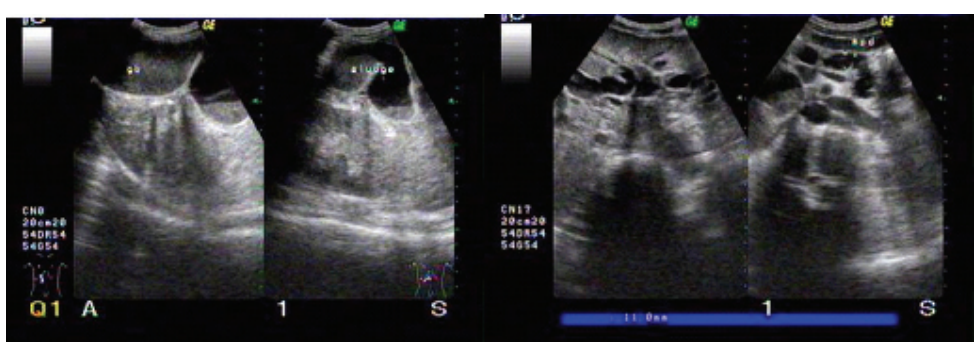

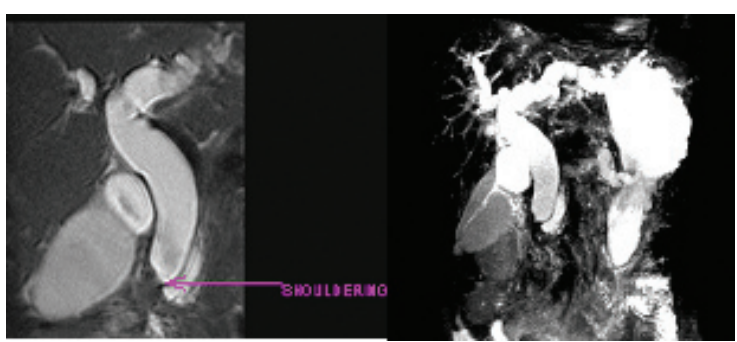

(b)

[Table/Fig-2]: A case of distal CBD stricture following repeated ERCP in a 60 yrs male who presented with features of gastric outlet obstruction (GOO) \& obstructive jaundice. (a) USG showed gross distention of the GB \& cystic duct (With intraluminal sludge), severe IHBR dilatation \& abrupt termination of distal CBD $(24 \mathrm{~mm})$. (b) MRCP confirmed the findings of USG. The abrupt termination of distal CBD is caused by complete narrowing in the ampullary region causing retrograde obstructive effects. Duodenum is compressed by the distended GB \& dilated CBD causing GOO. MPD is also dilated

evaluation of abdominal pain, if the diagnosis is uncertain. With these modalities the sensitivity for CBD stone diagnosis is low whereas specificity is higher than $90 \%[10,11]$.

Direct cholangiography is generally still considered to be the ideal method for CBD stone diagnosis. This has an added advantage of concurrent endoscopic sphincterotomy especially in a dilated CBD although ERCP may miss small stones. In one study with 28 consecutive cases of suspected CBD stones, the diagnosis was corroborated after surgical exploration in all cases. The study showed that $8.5 \%$ of the patients were false positive and underwent surgical exploration unnecessarily $[12,13]$. Thus the risk of surgical exploration related complications in patients with suspected CBD stones who ultimately are found to have no stones indicates the need for careful patient selection. A representative case of distal CBD stricture as sequelae of multiple ERCP is shown as [Table/Fig-2]. With the development of newer fast spin echo sequences without breath holding, significant improvement in image quality has been achieved. These imaging sequences provide excellent high T2 contrast with sharper delineation of the pancreaticobiliary tree anatomy $[14,15]$.

To improve the diagnostic accuracy for choledocholithiasis and stenosis (71 to 100), two dimensional maximum intensity projection image respiratory triggered TSE sequence technique (2D PACE) was performed in our study to avoid respiratory artifacts.

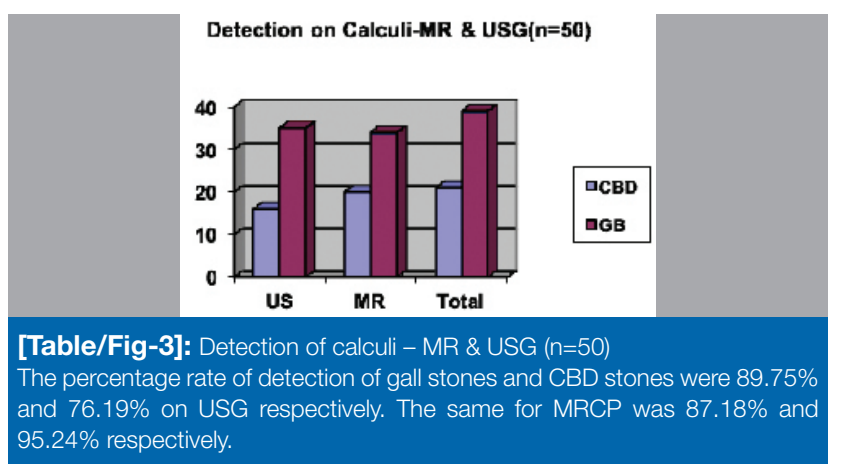

[15]. In our study, MRCP delineated normal and dilated CBDs in 27 patients (96\%).

With the development of higher magnetic field strength and newer pulse sequences, MRCP with its inherent high contrast resolution, rapidity, multiplanar capability and virtually artifact free display of anatomy and pathology in this region is proving to be examination of choice in patients with pancreatico biliary diseases [Table/Fig-2]. Few reports have specifically addressed the use of MRCP for diagnosing gallstone disease in children [16].

In this study of 50 patients with suspicion of gall stones \& CBD stones, 28 (70\%) actually had biliary stone disease, and 20 of these were diagnosed by MRCP. The study showed a $100 \%$ specificity but a much lower sensitivity for choledocholithiasis (56\%). This may be because patients with small CBD stones were missed by MRCP ( $6 \mathrm{~mm}$ ). Patients in the study were fasting before MRCP but did not receive any antiperistaltic agent, thus motion artifacts may have occurred during the acquisition time (almost two minutes); furthermore, small stones may be slowly stirred up in the CBD during the acquisition period. In addition, limitations of detection of small stones in maximum intensity projection images (MIP) are discussed subsequently.

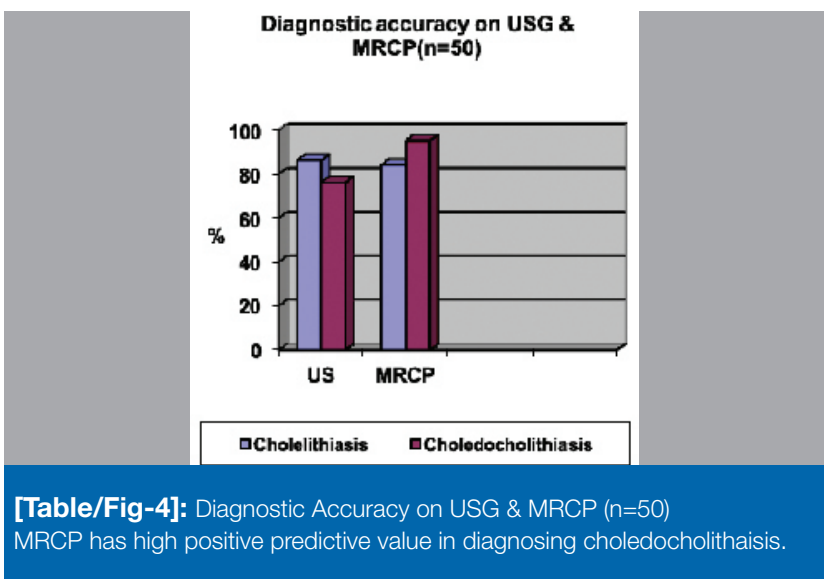




\begin{tabular}{|l|c|c|}
\hline & USG & MRCP \\
\hline Stones detected & $16(76 \%)$ & $20(95.5 \%)$ \\
\hline No stones & $5(24 \%)$ & $1(4.5 \%)$ \\
\hline Total & 21 & 21 \\
\hline
\end{tabular}

[Table/Fig-5]: Comparison of dilated CBD $(\mathrm{n}=28)$ diagnosis by Magnetic Resonance Cholangiopancreatography (MRCP) and reference methods The clinical role of MRCP in detection of dilated CBDs is higher as compared to the reference method (USG).

\begin{tabular}{|l|c|c|}
\hline & $\begin{array}{c}\text { No of cases observed } \\
\text { by reference method }\end{array}$ & $\begin{array}{c}\text { No of cases observed by } \\
\text { MRCP }\end{array}$ \\
\hline$\leq 6 \mathrm{~mm}$ & $5(31 \%)$ & $7(35 \%)$ \\
\hline$>6$ and $\leq 10 \mathrm{~mm}$ & $7(43 \%)$ & $9(45 \%)$ \\
\hline$>10 \mathrm{~mm}$ & $4(25 \%)$ & $4(20 \%)$ \\
\hline Total & 16 & 20 \\
\hline
\end{tabular}

[Table/Fig-6]: Choledocholithiasis diagnosis in relation to stone size.

\begin{tabular}{|c|c|c|c|}
\hline & $\begin{array}{c}\text { Dilated CBD } \\
\text { (reference } \\
\text { method) }\end{array}$ & $\begin{array}{l}\text { Undilated CBD } \\
\text { (reference } \\
\text { method) }\end{array}$ & Total \\
\hline Total & 28 & 22 & 50 \\
\hline $\begin{array}{l}\text { No stones (reference method)/ } \\
\text { no stones (MRC)-stones missed }\end{array}$ & $5 / 1$ & $2 / 1$ & $2 / 25$ \\
\hline \multicolumn{4}{|c|}{$\begin{array}{l}\text { [Table/Fig-7]: Choledocholithiasis: Diagnosis at MRCP correlated with } \\
\text { common bile duct (CBD) caliber. } \\
\text { MRCP was better investigative modality in detection of small pancreaticobiliary } \\
\text { calculi in dilated as well as normal undilated CBDs }\end{array}$} \\
\hline
\end{tabular}

\begin{tabular}{|l|c|c|c|c|c|}
\hline & USG & MRCP & $\begin{array}{c}\text { TOTAL } \\
\text { CASES }\end{array}$ & $\begin{array}{c}\text { Diagnostic } \\
\text { Accuracy } \\
\text { on USG }\end{array}$ & $\begin{array}{c}\text { Diagnostic } \\
\text { Accuracy } \\
\text { on MRCP }\end{array}$ \\
\hline Cholelithiasis & 35 & 34 & 39 & 86 & 84 \\
\hline Choledocholithiasis & 16 & 20 & 21 & 76 & 95 \\
\hline
\end{tabular}

\section{OTHER LESIONS}

\begin{tabular}{|l|c|c|c|c|c|}
\hline Cholangiocarcinoma & 2 & 3 & $3^{*}$ & 66 & 100 \\
\hline Pancreatitis & 4 & 6 & 8 & 50 & 75 \\
\hline $\begin{array}{l}\text { Periampullary } \\
\text { Carcinoma }\end{array}$ & 0 & 1 & $1^{\star}$ & - & 100 \\
\hline Carcinoma Pancreas & 2 & 3 & $4^{\star}$ & 50 & 75 \\
\hline $\begin{array}{l}\text { Post operative } \\
\text { Stricture }\end{array}$ & - & 2 & $2^{*}$ & - & 100 \\
\hline Choledochal Cyst & & 1 & $1^{*}$ & - & 100 \\
\hline Postoperative fistula & - & 1 & $1^{\star}$ & - & 100 \\
\hline Gall bladder mass & 1 & 1 & 1 & 100 & 100 \\
\hline
\end{tabular}

[Table/Fig-8]: Comparison of Diagnostic Accuracies of MRCP and USG in Pancreaticobiliary lesions: MRCP showed higher Diagnostic Accuracy in detection of Choledocholithiasis. However, USG results were comparable with MRCP for detection of Cholelithiasis.

In 21 cases of choledocholithiasis, diagnostic accuracy of ultrasound was $76 \%$. The distal CBD was difficult to visualize on ultrasound. MRI detected 26 cases of choledocholithiasis with a diagnostic accuracy of 95.5\% [Table/Fig-3,4].
Sensitivity and specificity of ultrasound and MRI were $73 \%$ and $89 \%$ and $97 \%$ and $98 \%$ [Table/Fig-5]. These data are comparable with that of Varghese et al., sensitivity $91 \%$, specificity $98 \%$ and diagnostic accuracy of 97\% (MRCP) [17]. In a similar study by Ke ZW et al., (2003), in evaluating the predictive value of MRCP in 267 patients before laparoscopic cholecystectomy (LC), MRCP had a sensitivity of $100 \%$, a specificity of $96.3 \%$, a positive predictive value of $91.8 \%$, and a negative predictive value of $100 \%$ for the detection of common bile duct stones [18].

Abdominal USG is the technique of choice for initial examination of the bile ducts, particularly in patients with jaundice.

MRCP is a noninvasive and non-operator-dependent technique that has a cost effective and important role in the evaluation of pancreaticobiliary diseases $[19,20]$. MRCP has higher diagnostic accuracy in demonstration of bile duct obstructions [Table/Fig-6], Choledocholithiasis, strictures and malignant obstructions [Table/Fig-, 7,8]. In our study, the Half-Fourier RARE MRCP produces high-quality images for delineation of the biliary and pancreatic ducts. The application of multiple echo trains and half-Fourier imaging reconstruction algorithms, half-Fourier RARE MRCP generates "fluidsensitive" images with negligible motion and susceptibility artifacts.

For the evaluation of postoperative complications, MRCP had high sensitivity for the depiction of strictures of anastomotic sites. Source images were better for evaluation of the morphology and length of the strictures. In our study, an anastomotic stricture was depicted with dilatation of the distal bile ducts and sudden abrupt termination at the anastomotic site.

Stones are easily recognized on MRCP images as low-signalintensity structures surrounded by high-signal-intensity bile. The smallest stone detected was $4 \mathrm{~mm}$ in diameter [Table/ Fig-5,8]. In our experience, small stones may have been obscured in maximum intensity projection images (MIP) as stones were likely obscured by the high signal intensity of the surrounding bile. The evaluation of basic source images was required to confirm the presence of calculi. In conclusion, halfFourier RARE MRCP exhibited excellent test performance and resulted in excellent inter-observer agreement for the evaluation of changes in patient's anatomy after pancreaticobiliary ductal system surgery. This imaging method is fast, safe, and noninvasive and can be used to follow-up patients, screen symptomatic patients for treatment, and help guide further therapeutic procedures [21].

The use of respiratory-triggered T2-weighted 2D PACE FSE MRCP sequence with parallel imaging, allowed high spatial resolution and relatively short acquisition times. This study has 
excellent sensitivity and specificity for possible biliary disease, specifically strictures, dilatation, and stones larger than $3 \mathrm{~mm}$, but is limited for stones $3 \mathrm{~mm}$ or smaller.

\section{CONCLUSION}

Our results present this study as a potential investigation of using MRCP in a tertiary hospital with standard equipment. MRCP has better diagnostic accuracy in detecting biliary calculi in cases of dilated and normal undilated CBD. The clinical role of MRCP in detection of dilated CBDs was also higher as compared to the reference method (USG) as shown in data of this study. We strongly recommend that MRCP be incorporated as a standard investigation in-patient with pancreaticobiliary gallstones for intervention and surgical planning. It is an effective cost effective tool and preoperative planning reduces the length of hospital stay.

\section{REFERENCES}

[1] Magnuson TH, Bender JS, Duncan MD. Utility of MR cholangiopancreatography in the evaluation of biliary obstruction. $J \mathrm{Am}$ Coll Surg.1999; 189: 63-72.

[2] Reinhold C, Taorel P, Bret P et al. Choledocholithiasis: evaluation of MR cholangiopancreatography for diagnosis. Radiology. 1998; 209:435-42.

[3] Baron RL. Common bile duct stones: reassessment criteria for ct diagnosis. Radiology. 1987; 162:419-24.

[4] Baron RL. Computed tomography of the biliary tree. RCNA 1991; 29(6): 1235-50.

[5] Dawson P, Adam A, Benjamin IS. Intravenous cholangiography revisited. Clinical Radiology.1993; 47:223-25.

[6] Patel JC, Bagely GS et al. The role of Intravenous cholangiography in pre operative assessment for laproscopic cholecystectomy. Br J Radiology. 1993; 66:1125-27.

[7] Wallener BK, Schumacher KA, Weidenmaier W, et al. Dilated biliary tract: evaluation with MR cholangiography with a T2 weighted contrast enhanced fast sequence. Radiology. 1991; 181:805-08.

[8] David et al: Pitfalls in the interpretation of MR cholangiopancreatography. AJR. 1998; 170:1055-59.

[9] Miyazaki T, Yamashita Y, Tsuchigame T, et al. MR cholangiopancreatography using HASTE (half-fourier acquisition single- shot turbo spin-echo) sequences. AJR Am J Roentgenol. 1996;166:1297-1303.

[10] Laubenberger $\mathrm{J}$ et al. Breath-hold projection MRCP: a new method for examination of the bile and pancreatic ducts. Magn Reson Med. 1995; 33:1823.

[11] David $V$ et al: Pitfalls in the interpretation of MR cholangiopancreatography. AJR. 1998; 170:1055-59.

[12] Prat F, Bou Jaoude J, Ink O, et al. Endoscopic sphincterotomy for suspected choledocholithiasis in patients with and without stones. Am J Gastroenterol. 1995;90:727-31.

[13] Lightdale CJ. Indications, contraindications and complications of endoscopic ultrasonography. Gastrointest Endosc 1996;43:S15-18.

[14] Outwater E, Miller L, Gordon S, et al. Non invasive 3-d cholangiography with MR imaging. [Abstract]. Gastroenterology. 1994:106:A374 .

[15] Guibaud L, Bret PM, Reinhold C, et al. Bile duct obstruction and choledocholithiasis: diagnosis with MR cholangiography. Radiology. 1995;197:109-15.

[16] Fitoz S, Erden A, Boruban S. Magnetic resonance cholangiopancreatography of biliary system abnormalities in children. Clin Imaging. 2007;31(2):93-101.

[17] Varghese JC, Liddell RP, Farrell MA, Murray FE, Osborne $\mathrm{DH}$, Lee MJ. Diagnostic accuracy of magnetic resonance cholangiopancreatography and ultrasound compared with direct cholangiography in the detection of choledocholithiasis. Clin Radiol. 2000;55(1):25-35. Erratum in: Clin Radiol 2000; 55(8):657.

[18] Ke ZW, Zheng CZ, Li JH, Yin K, Hua JD. Prospective evaluation of magnetic resonance cholangiography in patients with suspected common bile duct stones before laparoscopic cholecystectomy. Hepatobiliary Pancreat Dis Int. 2003;2(4):576-80.

[19] Zhang C, Yin M, Liu Q.The Guidance impact of preoperative magnetic resonance cholangiopancreatography on laparoscopic cholecystectomy. J Laparoendosc Adv Surg Tech A. 2015 Aug 11. [Epub ahead of print].

[20] Ward WH, Fluke LM, Hoagland BD, Zarow GJ, Held JM, Ricca $\mathrm{RL}$. The role of magnetic resonance cholangiopancreatography in the diagnosis of choledocholithiasis: do benefits outweigh the costs? Am Surg. 2015;81(7):720-25.

[21] Lin C, Collins JN, Britt RC, Britt LD. Initial cholecystectomy with cholangiography decreases length of stay compared to preoperative MRCP or ERCP in the management of choledocholithiasis. Am Surg. 2015 ;81(7):726-31.

\section{AUTHOR(S):}

1. Dr. SN Singh

2. Dr. Trilok C Bhatt

\section{PARTICULARS OF CONTRIBUTORS:}

1. Classified Specialist (Radiodiagnosis) MD Radiodiagnosis, Department of Radiology, INHS Kalyani, Gandhigram Po Visakhapatnam, India.

2. Graded Specialist (Radiodiagnosis), MD Radiodiagnosis, Department of Radiology, INHS Kalyani, Gandhigram Po Visakhapatnam, India.

\section{NAME, ADDRESS, E-MAIL ID OF THE CORRESPONDING AUTHOR:}

Dr. SN Singh,

MD Radiology, INHS Kalyani

Gandhigram Post Vishakhapatnam-53005, India.

E-mail: cdrsingh@gmail.com

FINANCIAL OR OTHER COMPETING INTERESTS: None. Date of Publishing: Jan 05, 2016 